\title{
150 Hefte GW-Unterricht - ein erweitertes Editorial
}

\author{
G. K. Lieb, A. Koller, C. Fridrich, T. Jekel, L. Keller, A. Oberrauch \& H. Pichler
}

Runde Zahlen werden seit jeher als etwas Besonderes wahrgenommen und, wenn sie in zeitlichen Abläufen auftreten, etwa als runde Geburtstage oder Jubiläen gefeiert. GW-Unterricht hätte dazu im laufenden Jahr 2018 doppelt Grund, denn die Zeitschrift gibt es nunmehr bereits seit 40 Jahren und das Heft, das Sie in der Hand halten, ist die 150. Ausgabe. Gleich vorweg: Feierlichkeiten sind nicht vorgesehen - das Redaktionsteam konzentriert sich lieber auf die Herausforderungen der laufenden Arbeit. Ganz unbemerkt vorbeigehen lassen wollen wir das Jubiläum aber auch nicht!

So sei, wie bei Anlässen dieser Art üblich, mit einem kurzen Rückblick begonnen. Unsere Zeitschrift wurde auf Initiative des Doyens der modernen GW-Didaktik, Wolfgang Sitte (1925-2006) ins Leben gerufen und erschien unter der Redaktion von Gerhard Atschko, Wolfgang Sitte und Ernst Weber erstmals im September 1978. Das Erscheinen nicht nur dieses ersten Heftes, sondern aller weiteren in den folgenden rund drei Jahrzehnten wurde durch die Zentralsparkasse der Gemeinde Wien (die Z) und die spätere Bank Austria-Creditanstalt (BA-CA) finanziert. Dies geschah vor dem Hintergrund, dass diese Banken Interesse an der ökonomischen Bildung der Schüler/innen, damals „Spar- und Wirtschaftserziehung“ genannt, hatten und auf diese Weise die Lehrer/innen unterstützen wollten. Details zu diesen frühen Entwicklungen finden sich in den Beiträgen von Atschko (2005) und Pirker (2005). Beide erschienen in der 100. Ausgabe von GW-Unterricht, die gleichzeitig auch eine Festschrift zum 80. Geburtstag von W. Sitte war. Brigitte Pirker von der BA-CA, bald schon Mitglied im Redaktionsteam, war für lange Jahre die Schlüsselperson in der Kooperation mit diesem Kreditinstitut und der ,gute Geist' dieser Zeitschrift.

Daraus geht schon hervor, dass sich die Zeitschrift in der ,Szene' rasch etablierte, füllte sie doch eine wichtige Lücke als jenes Publikationsorgan, das die spezifischen Ansprüche der seit 1962 verankerten Zusammenführung der Wissenschaftsdisziplinen Geographie und Ökonomie im Unterrichtsfach Geographie \& Wirtschaftskunde erfüllte (vgl. Sitte 2006). Die Zeitschrift enthielt von Anfang an Beiträge zur Fachdidaktik, zur Fachwissenschaft, zur Unterrichtspraxis und zu Service - wie die entsprechenden Rubriken auch heute heißen - und bediente damit sowohl den fachdidaktisch-schulgeographischen Diskurs (schon im ersten Heft: Sitte 1978) als auch die Bedürfnisse der Lehrpersonen in den Schulen. Ein besonderes Anliegen blieb - über die wechselnden Redaktionsteams hinweg - bis heute die sinnvolle Verbindung des , $\mathrm{G}^{\boldsymbol{}}$ und des , $\mathrm{W}^{`}$, sodass Schüler/innen beim Erwerb alltagsrelevanter Kompetenzen (wie man heute wohl sagen würde) unterstützt werden. Dabei entwickelte sich unsere Zeitschrift, worauf wir als Redaktionsteam besonders stolz sind, auch zu einer Plattform zur Aushandlung diskursiver Themen in der GW-Didaktik, was sowohl an den Beiträgen aus unterschiedlichen fachdidaktischen Paradigmen als auch an der Beitragskategorie ,Kontrapunkt erkennbar wird.

Die Produktion der Zeitschrift wurde mit der Zeit immer professioneller, was sich auch im veränderten, mehrfach dem Zeitgeschmack angepassten Layout widerspiegelt. Dies sollen die verschiedenen Generationen des Erscheinungsbildes auf der Titelseite des vorliegenden Heftes exemplarisch zeigen. Indirekt symbolisieren sie auch die wohl markanteste Veränderung in der Geschichte von GW. 2007 wurde Realität, was sich schon länger abgezeichnet hatte. Die BA-CA finanzierte GW-Unterricht - ebenso wie die mitunter als legendär konnotierten Fachdidaktiktreffen am Haimingerberg (Tirol) - nicht mehr, was bedeutete, dass nunmehr andere finanzielle Quellen für das Erscheinen der Zeitschrift gefunden werden mussten.

Durch den gänzlichen Wegfall dieses Sponsors mussten durch ein neues Redaktionsteam Finanzierung, Redaktionsabläufe und Publikationsweise völlig neu gestaltet werden. Die für elektronische Dienstleistungen, Lektorat, Layout, Druck und Werbung erforderlichen Mittel mussten durch Inserate, Medienpartnerschaften und in jüngster Zeit durch Open-Access-Gebühren gestellt werden. Nur am Rande sei erwähnt, dass alle Redaktionsmitglieder ehrenamtlich für diese Zeitschrift arbeiten. Die komplette Redaktionsarbeit wird seitdem von der Einreichung über die Begutachtung bis hin zur Endproduktion über eine Plattform abgewickelt. 
Seit 2009 erscheint GW-Unterricht viermal jährlich nicht nur in gedruckter Form, sondern auch online, seit 2014 in einer Open-Access-Lizenz beim Verlag der Österreichischen Akademie der Wissenschaften. Auch über die Homepage www.gw-unterricht.at sind die Artikel erreichbar. Im Zuge dieser Umstellungen kam es auch zur Etablierung des neuen, nunmehr von (fast) allen österreichischen Universitäten und Pädagogischen Hochschulen mit GW-Ausbildung beschickten Redaktionsteam, das nun die Geschicke der Zeitschrift leitet. Zu den wichtigen Errungenschaften der jüngeren Zeit zählen die Aufnahme in die Liste der anerkannten wissenschaftlichen Zeitschriften nach dem Verband der Geographen an deutschen Hochschulen (VdGH) 2014 und die Registrierung im Directory of Open Access Journals (DOAJ). Eine Voraussetzung dafür war das schon einige Jahre zuvor eingeführte, für alle Beiträge in den Kategorien Fachwissenschaft, Fachdidaktik und Unterrichtspraxis obligate, redaktionsexterne Double-blind-Peer-Review-Verfahren.

Dies dient der Qualitätssicherung bzw. -steigerung unserer Zeitschrift, die auch in Zukunft ein zentrales Credo unserer Arbeit bleiben werden. Stichwort Zukunft: Wir können einen optimistischen Blick dorthin richten, denn das Redaktionssystem ist in der Regel mit eingereichten Manuskripten gut gefüllt und die Finanzierung erscheint gesichert. Dennoch: Die Zahl der Beiträge könnte durchaus größer werden und ganz allgemein würde auch mehr Feedback - speziell aus der Unterrichtspraxis - hilfreich sein. Besonders freuen uns dabei die Stimmen jener, die mit jungen und frischen Ideen entweder die Unterrichtspraxis oder wissenschaftliche Fachdidaktik betreten, und uns dabei helfen in Bewegung zu bleiben. Unterstützen Sie uns also bitte dabei, dass wir eine noch gewichtigere Stimme für unser Fach werden, denn so manche gesellschaftliche und bildungspolitische Entwicklung legt nahe, dass dies in Zukunft noch bedeutender werden könnte, als es ohnehin schon ist.

\section{Literatur}

Atschko G. (2005): 27 Jahre - 100 mal GW-UNTERRICHT - 12 mal GW-KOMPAKT. In: GW-Unterricht 100, 9-11. Pirker B. (2005): Ehre, wem Ehre gebührt. In: GW-Unterricht 100, 2-8 .

Sitte W. (1978): Zur gegenwärtigen Situation des GW-Unterrichts in Österreich. In: GW-Unterricht 1, 1-4.

Sitte W. (2006): Geographie und Wirtschaftskunde (GW) - Entwicklung und Konzept des Unterrichtsfachs. In: Sitte, W., Wohlschlägl, H. (Hg.): Beiträge zur Didaktik des „Geographie und Wirtschaftskunde“-Unterrichts. Wien: Materialien zur Didaktik der Geographie und Wirtschaftskunde, Bd. 16, 157-169.

\section{Zu diesem Heft}

Eine dieser aktuellen Entwicklungen ist die Kritik an der Qualität der ökonomischen Bildung im Rahmen des GW-Unterrichts. Zahlreiche Beiträge unserer Zeitschrift haben sich in den letzten Jahren damit auseinandergesetzt, und auch in diesem Heft sind die beiden Artikel in der Rubrik Fachdidaktik diesem Diskurs zuzuordnen. Anke Uhlenwinkel erforscht die Validität zweier empirischer Studien, durch die versucht wurde, die mangelnde Fähigkeit von GW-Lehrer/inn/en zum Unterrichten wirtschaftlicher Inhalte zu belegen. Sie kommt zu einem sehr kritischen Ergebnis und betont das Potential der Basiskonzepte (key concepts). Sandra Stieger und Thomas Jekel untersuchen in einem weiteren Beitrag die Interessen und die impliziten Annahmen, die hinter neun empirischen Studien zum Wirtschaftswissen österreichischer Schüler/innen stecken, aus dem Blickwinkel ihrer wissenschaftlichen und institutionellen Einbettung sowie den aus den empirischen Befunden abgeleiteten politischen Empfehlungen.

Der in der Rubrik Unterrichtspraxis eingeordnete Artikel von Matthias Kowasch und einem Team von Autor/ inn/en entwickelt und begründet ein Unterrichtsmodell, durch welches die Mehrdimensionalität von Konsumhandlungen für Schüler/innen beispielhaft erfahrbar gemacht und der „klassische“ Konsumansatz unter dem Aspekt der Bildung für Nachhaltige Entwicklung dekonstruiert wird. Dieses Unterrichtsbeispiel wurde im Rahmen der Fachtagung „Zukunft Fachdidaktik GW 2017“ in Schlierbach entwickelt und belegt somit, dass aus dieser Veranstaltung auch konkrete Produkte für die Unterrichtspraxis entstehen. Ihre heurige "Neuauflage“ stand unter dem Motto "Zwischen Fake News und Unterrichtsmaterial - Medien in der geographischen und wirtschaftlichen Bildung", worüber Herbert Pichler und Matthias Fasching berichten.

Möge die Lektüre dieses Heftes viele Anregungen und Denkanstöße für Sie bringen! 